\title{
Clinical Characteristics in Patients with Triple Negative Breast Cancer
}

\author{
Janet Yeh,, Jennifer Chun, ${ }^{1}$ Shira Schwartz, ${ }^{1}$ Annie Wang, ${ }^{2}$ Elizabeth Kern, ${ }^{3}$ \\ Amber A. Guth, ${ }^{1}$ Deborah Axelrod, ${ }^{1}$ Richard Shapiro, ${ }^{1}$ and Freya Schnabel ${ }^{1}$ \\ ${ }^{1}$ Department of Surgery, New York University Langone Medical Center, New York, NY, USA \\ ${ }^{2}$ School of Medicine, New York University Langone Medical Center, New York, NY, USA \\ ${ }^{3}$ Drexel University, School of Medicine, Philadelphia, PA, USA
}

Correspondence should be addressed to Freya Schnabel; freya.schnabel@nyumc.org

Received 15 February 2017; Accepted 16 July 2017; Published 17 August 2017

Academic Editor: Debra A. Tonetti

Copyright (C) 2017 Janet Yeh et al. This is an open access article distributed under the Creative Commons Attribution License, which permits unrestricted use, distribution, and reproduction in any medium, provided the original work is properly cited.

Purpose. The purpose of this study was to compare and contrast the clinical characteristics of the triple negative breast cancer (TNBC) and non-TNBC patients, with a particular focus on genetic susceptibility and risk factors prior to diagnosis. Methods. Our institutional database was queried for all patients diagnosed with invasive breast cancer between January 2010 and May 2016. Results. Out of a total of 1964 patients, 190 (10\%) patients had TNBC. The median age for both TNBC and non-TNBC was 59 years. There was a significantly higher proportion of African American and Asian patients with TNBC $(p=0.0003)$ compared to patients with non-TNBC. BRCA1 and BRCA2 were significantly associated with TNBC $(p<0.0001, p=0.0007)$. A prior history of breast cancer was significantly associated with TNBC $(p=0.0003)$. There was no relationship observed between TNBC and a history of chemoprevention or patients who had a history of AH or LCIS. Conclusions. We found that having Asian ancestry, a prior history of breast cancer, and a BRCA1 or BRCA2 mutation all appear to be positively associated with TNBC. In order to develop more effective treatments, better surveillance, and improved prevention strategies, it is necessary to improve our understanding of the population at risk for TNBC.

\section{Introduction}

Triple negative breast cancer (TNBC) is the subtype of breast cancer that does not overexpress human epidermal growth factor 2 receptors (HER2), while also lacking expression of estrogen receptors (ER) and progesterone receptors (PR). TNBC, which accounts for an estimated $15-20 \%$ of invasive breast cancers [1-3], has been associated with rapid growth, distant metastasis, and shorter overall and relapse-free survival when compared to other breast cancer subtypes across multiple studies [4-6].

Much of the literature surrounding TNBC has been focused on identifying populations at risk. In particular, BRCA1 and BRCA2 genotypes have been shown to predispose carriers to TNBC [5,7-11]. The BRCA genes encode tumor suppressors that repair DNA damage by homologous recombination; when mutated, the carrier is susceptible to breast and ovarian cancer [12]. Studies suggest 10.6-30.9\% of TNBC patients are carriers for deleterious BRCA1 and BRCA2 germline mutations, especially BRCA1 [7, 10, 13, 14]. Routine BRCA mutation testing is not recommended for all patients with breast cancer due to its high cost and the low prevalence of mutations [15-17]. However, the National Comprehensive Cancer Network (NCCN) guidelines recommend referral for consideration of genetic counseling for women 60 years of age or younger with TNBC $[13,18]$. Recent reports have also shown strong associations between TNBC and diagnosis of breast cancer at a younger age $[19,20]$.

Another population with increased incidence of TNBC is African American patients [1,21-23]. Carey et al. devised a case-control study in North Carolina in which African American patients were overrepresented to allow for statistical comparison to mostly Caucasian patients; this landmark study found that $39 \%$ of African American patients presented with TNBC, compared to $16 \%$ of non-African American patients [1]. Amirikia and colleagues analyzed the prevalence 
of TNBC among non-Hispanic White, non-Hispanic Black, and Hispanic patients in the California Cancer Registry; they confirmed the association and asserted a steeper rise in incidence of TNBC with age among African American women [21]. Multiple locoregional studies demonstrate that TNBC comprises 20-40\% studies of African American breast cancer patients $[1,21-24]$. However, there have not been any American studies analyzing TNBC in the Asian population. Among 972 breast cancer patients at the Dr. B. Borooah Cancer Institute in India, 31.9\% were defined as TNBC [25]. Additionally, the University Malaya Medical Center in Malaysia reports a $17.6 \%$ incidence of TNBC among 1147 breast cancer patients of Chinese, Malay, and Indian descent [26]. From these international reports, we suspect that TNBC may affect a large proportion of Asian American patients.

The present study was designed to compare and contrast the clinical characteristics of the TNBC and non-TNBC patients in the New York University Breast Cancer Database (BCD), with a particular focus on genetic susceptibility and risk factors prior to cancer diagnosis.

\section{Methods}

2.1. Study Participants. The Breast Cancer Database (BCD) is a longitudinal study that was established at our institution in January 2010. All patients undergoing definitive breast cancer surgery for a newly diagnosed breast cancer at our institution are eligible to enroll in the BCD. The variables collected for the database include information on personal and family history, screening history, methods of diagnosis, tumor histology and stage at diagnosis, details of treatment, and outcomes. All clinical data are obtained from detailed questionnaires that are filled out at the time of surgery and from a review of electronic medical records. The Breast Cancer Database was queried for all patients who were diagnosed with invasive breast cancer between January 2010 and May 2016. This study was approved by the Institutional Review Board.

2.2. Statistical Analyses. Descriptive statistics were used to summarize the data and to see the distribution of the variables between patients with TNBC and patients with non-TNBC. The variables of interest included age, race, personal and family history of breast cancer, BRCA1 and BRCA2 status, and tumor characteristics. Pearson's chi-square and Fisher's exact tests were used to assess any associations between the categorical variables of interest and TNBC status with a significance level of 0.05 . Logistic regression was used to test for any associations between the continuous predictor variables, age and tumor size, and TNBC. All analyses were performed using SAS version 9.3 (SAS Institute Inc., Cary, NC).

\section{Results}

During the study period, a total of 1964 patients with invasive breast cancer were enrolled in the Breast Cancer Database. The majority of patients in the study cohort were Caucasian (76\%). The median age was 59 years (22-95 years). There were 31 (5\%) patients who were BRCA1 positive and 29 (5\%) who were BRCA2 positive. As expected, the majority of breast cancer patients had tumors that were early stage, stage I or II, (91\%) and of ductal histology (81\%).

Out of a total of 1964 patients, 190 (10\%) patients had TNBC and $1774(90 \%)$ had non-TNBC. The median age for both TNBC and non-TNBC was 59 years. There was a significantly higher proportion of African American and Asian patients with TNBC $(p=0.0003)$ compared to patients with non-TNBC (Table 1). BRCA1 and BRCA2 were significantly associated with TNBC $(p<0.0001, p=0.0007)$. A prior history of breast cancer was significantly associated with triple negative histology in our study $(p=0.0003)$. There was no relationship observed between TNBC status and a history of chemoprevention. Likewise, there was no relationship between TNBC status and patients who had a history of AH or LCIS (Table 1). When compared to patients with non-TNBC, patients with TNBC were more likely to have undergone neoadjuvant treatment $(p<0.0001)$. As compared to patients with non-TNBC, patients with TNBC were also more likely to have later stage disease $(p=0.0001)$, invasive ductal carcinomas $(p<0.0001)$, and higher Ki-67 $(p<0.0001)$ (Table 2).

\section{Discussion}

The results of our study both confirm known associations of TNBC and identify significant notable findings specific to our TNBC population. Similar to findings in previous studies, our TNBC patients had an increased proportion of African American patients and a higher percentage of BRCA mutation carriers when compared to the non-TNBC population. A significant association between TNBC and previous history of breast cancer was also seen. No association was found between TNBC and prior history of atypical hyperplasia or LCIS. Contrary to many previously published studies, we found a higher proportion of Asians with TNBC. We also found that the median age in both TNBC and non-TNBC cohorts was 59 years.

TNBC has been shown to be associated with a 10.6-30.9\% carrier rate of deleterious BRCA1 and BRCA2 mutations $[7,10,13,14]$. Our data is consistent with an increased number of carriers in our TNBC population. This may be due to the unique racial distribution of our patients. Internationally, there are differences in BRCA1 and 2 prevalence; for example, among 190 TNBC patients in Mexico City, 23\% were found to have a founder BRCA1 mutation (BRCA1 ex9-12del) [27], while BRCA risk calculators developed in Caucasian populations consistently underestimate BRCA risk in Asian patients [28]. We postulate that the higher proportion of Asian American patients in our database may account for this discrepancy with previous literature. The NCCN guidelines recommend that all women with TNBC and 60 years of age or younger should undergo genetic counseling [18]; moreover, our study supports the NCCN guideline and underlines the importance of genetic counseling for TNBC patients.

There was a significant association between TNBC and individuals of African American and Asian heritage within our cohort. We identified $18 \%$ and $13 \%$ of individuals in the 
TABLE 1: Clinical characteristics.

\begin{tabular}{|c|c|c|c|c|c|}
\hline Variables & TNBC $(N=190,10 \%)$ & $\%$ & Non-TNBC $(N=1774,90 \%)$ & $\%$ & $p$ value \\
\hline Median age, in years & $59(25-92$ & & $59(22-95)$ & & 0.52 \\
\hline \multicolumn{6}{|l|}{ Race } \\
\hline African American & 31 & 16 & 147 & 8 & \multirow{5}{*}{0.0003} \\
\hline Asian & 23 & 12 & 152 & 9 & \\
\hline White & 122 & 64 & 1363 & 77 & \\
\hline Hispanic & 11 & 6 & 103 & 6 & \\
\hline Other & 3 & 2 & 9 & 1 & \\
\hline \multicolumn{6}{|l|}{$A H$} \\
\hline No & 188 & 99 & 1741 & 98 & \multirow{2}{*}{0.57} \\
\hline Yes & 2 & 1 & 33 & 2 & \\
\hline \multicolumn{6}{|l|}{ LCIS } \\
\hline No & 190 & 100 & 1751 & 99 & \multirow{2}{*}{0.16} \\
\hline Yes & 0 & 0 & 23 & 1 & \\
\hline \multicolumn{6}{|c|}{ Previous history of breast cancer } \\
\hline No & 156 & 82 & 1605 & 90 & \multirow{2}{*}{0.0003} \\
\hline Yes & 34 & 18 & 169 & 10 & \\
\hline \multicolumn{6}{|c|}{ Family history of breast cancer } \\
\hline No & 144 & 76 & 1352 & 76 & \multirow{2}{*}{0.90} \\
\hline Yes & 46 & 24 & 442 & 24 & \\
\hline \multicolumn{6}{|l|}{$B R C A 1$} \\
\hline Negative & 65 & 82 & 481 & 97 & \multirow{3}{*}{$<0.0001$} \\
\hline Positive & 14 & 18 & 17 & 3 & \\
\hline Unknown/not tested & 111 & - & 1276 & - & \\
\hline \multicolumn{6}{|l|}{$B R C A 2$} \\
\hline Negative & 68 & 86 & 480 & 96 & \multirow{3}{*}{0.0007} \\
\hline Positive & 11 & 14 & 18 & 4 & \\
\hline Unknown/not tested & 111 & - & 1276 & - & \\
\hline \multicolumn{6}{|l|}{ Chemoprevention } \\
\hline No & 179 & 94 & 1695 & 96 & \multirow{2}{*}{0.40} \\
\hline Yes & 11 & 6 & 79 & 4 & \\
\hline
\end{tabular}

TABle 2: Tumor characteristics.

\begin{tabular}{|c|c|c|c|c|c|}
\hline Variables & $\operatorname{TNBC}(N=190,10 \%)$ & $\%$ & Non-TNBC $(N=1774,90 \%)$ & $\%$ & $p$ value \\
\hline \multicolumn{6}{|l|}{ Neoadjuvant } \\
\hline No & 157 & 83 & 1669 & 94 & \multirow{2}{*}{$<0.0001$} \\
\hline Yes & 33 & 17 & 105 & 6 & \\
\hline Median tumor size $(\mathrm{cm})$ & \multicolumn{2}{|c|}{$1.6(0.1-9.4)$} & \multicolumn{2}{|c|}{$1.3(0.04-12.5)$} & 0.06 \\
\hline \multicolumn{6}{|l|}{ Stage } \\
\hline I & 101 & 53 & 1111 & 63 & \multirow{4}{*}{0.0001} \\
\hline IIA, IIB & 55 & 29 & 513 & 29 & \\
\hline IIIA, IIIB, IIIC & 33 & 17 & 138 & 8 & \\
\hline IV & 1 & 1 & 12 & 1 & \\
\hline \multicolumn{6}{|l|}{ Histology } \\
\hline IDC & 178 & 94 & 1422 & 80 & \\
\hline ILC & 5 & 3 & 242 & 14 & $<0.0001$ \\
\hline Invasive other & 7 & 4 & 110 & 6 & \\
\hline Median Ki-67 & \multicolumn{2}{|c|}{60 (1-99) } & \multicolumn{2}{|c|}{$10(1-99)$} & $<0.0001$ \\
\hline
\end{tabular}


TNBC population as African American and Asian, respectively. African American and Asian patients each comprise $8 \%$ of the non-TNBC population. The association between African American patients and TNBC is well established in several studies [1, 21-23]. However, there is insufficient data on the association of TNBC with individuals of Asian descent in current medical literature. There may be distinct Asian populations that are more susceptible to TNBC than others, which would explain our findings. Jack et al. showed a similar association between TNBC and a South Asian population [29], while Lakshmaiah et al. showed a higher incidence of TNBC in an Indian population compared to Western populations [30]. Thus, further assessments should be performed to differentiate Asian populations with increased associations with TNBC.

The average age of diagnosis for TNBC has been shown to be 5-10 years younger than patients with non-TNBC [11]. Of note, the median age was the same in our TNBC and non-TNBC cohorts. The older median age of our TNBC patients may be related to the increase of patients with a prior personal history of breast cancer and may be attributed to the prior use of endocrine therapy. It has been shown that endocrine therapy reduces the risk of ER positive breast cancer [31-33]. On long-term follow-up, the likelihood of TNBC is shown to increase. However, we were not able to demonstrate a relationship between having TNBC and prior use of endocrine therapy in our cohort. This may be due to low numbers of patients with a prior history of chemoprevention.

$\mathrm{AH}$ and LCIS are known global risk factors linked to the development of breast cancer in women [34-36]. Our study confirmed a lack of association between TNBC and a prior history of AH or LCIS. This may be because most women with $\mathrm{AH}$ and LCIS decline chemoprevention and a large proportion of patients who start chemoprevention do not complete the course due to side effects $[37,38]$. Therefore, patients with a prior history of AH and LCIS have lower exposure to endocrine therapies than those with prior history of breast cancer. Additionally, it has been theorized that there may be a different pathogenesis of TNBC compared to other subtypes of breast cancer [39].

\section{Conclusions}

While our study confirmed known characteristics associated with TNBC, it also highlighted unexpected results that merit further investigation. Based on our TNBC population, we found that having Asian ancestry, a previous personal history of breast cancer, or a germline BRCA mutation all appear to be positively associated with TNBC. This finding further supports the revised NCCN guidelines that recommend women 60 years of age or younger with TNBC to be referred for consideration of genetic counseling. In addition, there was a lack of association between TNBC and personal history of $\mathrm{AH}$ and LCIS.

In order to develop more effective treatments, better surveillance, and improved prevention strategies, it is critical to improve our understanding of the risk factors that are associated with the development of triple negative breast cancer.

\section{Conflicts of Interest}

The authors declare that they have no conflicts of interest.

\section{References}

[1] L. A. Carey, C. M. Perou, C. A. Livasy et al., "Race, breast cancer subtypes, and survival in the Carolina Breast Cancer Study," The Journal of the American Medical Association, vol. 295, no. 21, pp. 2492-2502, 2006.

[2] P. L. Nguyen, A. G. Taghian, M. S. Katz et al., "Breast cancer subtype approximated by estrogen receptor, progesterone receptor, and HER-2 is associated with local and distant recurrence after breast-conserving therapy," Journal of Clinical Oncology, vol. 26, no. 14, pp. 2373-2378, 2008.

[3] T. Sørlie, "Molecular portraits of breast cancer: Tumour subtypes as distinct disease entities," European Journal of Cancer, vol. 40, no. 18, pp. 2667-2675, 2004.

[4] T. Sørlie, C. M. Perou, and R. Tibshirani, "Gene expression patterns of breast carcinomas distinguish tumor subclasses with clinical implications," Proceedings of the National Academy of Sciences of the United States of America, vol. 98, no. 19, pp. 1086910874, 2001.

[5] T. Sørlie, R. Tibshirani, and J. Parker, "Repeated observation of breast tumor subtypes in independent gene expression data sets," Proceedings of the National Academy of Sciences of the United States of America, vol. 100, pp. 8418-8423, 2003.

[6] N. U. Lin, A. Vanderplas, M. E. Hughes et al., "Clinicopathologic features, patterns of recurrence, and survival among women with triple-negative breast cancer in the National Comprehensive Cancer Network," Cancer, vol. 118, no. 22, pp. 5463-5472, 2012.

[7] R. Greenup, A. Buchanan, W. Lorizio et al., "Prevalence of BRCA mutations among women with triple-negative breast cancer (TNBC) in a genetic counseling cohort," Annals of Surgical Oncology, vol. 20, no. 10, pp. 3254-3258, 2013.

[8] E. H. Lips, L. Mulder, A. Oonk et al., "Triple-negative breast cancer: BRCAness and concordance of clinical features with BRCA1-mutation carriers," British Journal of Cancer, vol. 108, no. 10, pp. 2172-2177, 2013.

[9] G. Palomba, M. Budroni, N. Olmeo et al., "Triple-negative breast cancer frequency and type of BRCA mutation: Clues from Sardinia," Oncology Letters, vol. 7, no. 4, pp. 948-952, 2014.

[10] P. Sharma, J. R. Klemp, B. F. Kimler et al., "Germline BRCA mutation evaluation in a prospective triple-negative breast cancer registry: Implications for hereditary breast and/or ovarian cancer syndrome testing," Breast Cancer Research and Treatment, vol. 145, no. 3, pp. 707-714, 2014.

[11] L. A. Newman, J. S. Reis-Filho, M. Morrow, L. A. Carey, and T. A. King, "The 2014 Society of Surgical Oncology Susan G. Komen for the Cure Symposium: Triple-Negative Breast Cancer," Annals of Surgical Oncology, vol. 22, no. 3, pp. 874-882, 2015.

[12] J. L. Boerner, N. Nechiporchik, K. L. Mueller et al., "Protein expression of DNA damage repair proteins dictates response to topoisomerase and PARP inhibitors in triple-negative breast cancer," PLoS ONE, vol. 10, no. 3, Article ID e0119614, 2015.

[13] A.-R. Hartman, R. R. Kaldate, L. M. Sailer et al., "Prevalence of BRCA mutations in an unselected population of triple-negative breast cancer," Cancer, vol. 118, no. 11, pp. 2787-2795, 2012.

[14] A. M. Gonzalez-Angulo, K. M. Timms, S. Liu et al., "Incidence and outcome of BRCA mutations in unselected patients with 
triple receptor-negative breast cancer," Clinical Cancer Research, vol. 17, no. 5, pp. 1082-1089, 2011.

[15] J. Balmaña, O. Díez, I. T. Rubio, and F. Cardoso, "BRCA in breast cancer: ESMO clinical practice guidelines," Annals of Oncology, vol. 22, no. 6, pp. vi31-vi34, 2011.

[16] J. S. Kwon, A. M. Gutierrez-Barrera, D. Young et al., "Expanding the criteria for BRCA mutation testing in breast cancer survivors," Journal of Clinical Oncology, vol. 28, no. 27, pp. 42144220, 2010.

[17] J. D. Fackenthal and O. I. Olopade, "Breast cancer risk associated with BRCA1 and BRCA2 in diverse populations," Nature Reviews Cancer, vol. 7, no. 12, pp. 937-948, 2007.

[18] NCCN Clinical Practice Guidelines in Oncology (NCCN Guidelines) Genetic/Familial High-Risk Assessment: Breast and Ovarian, (ed 4.2013), National Comprehensive Cancer Network.

[19] K. R. Bauer, M. Brown, R. D. Cress, C. A. Parise, and V. Caggiano, "Descriptive analysis of estrogen receptor (ER)negative, progesterone receptor (PR)-negative, and HER2negative invasive breast cancer, the so-called triple-negative phenotype," Cancer, vol. 109, no. 9, pp. 1721-1728, 2007.

[20] P. Boyle, "Triple-negative breast cancer: epidemiological considerations and recommendations," Annals of Oncology, vol. 23, no. 6, Article ID mds187, pp. vi7-vil2, 2012.

[21] K. C. Amirikia, P. Mills, J. Bush, and L. A. Newman, "Higher population-based incidence rates of triple-negative breast cancer among young African-American women. Implications for breast cancer screening recommendations," Cancer, vol. 117, no. 12, pp. 2747-2753, 2011.

[22] M. J. Lund, K. F. Trivers, P. L. Porter et al., "Race and triple negative threats to breast cancer survival: A population-based study in Atlanta, GA," Breast Cancer Research and Treatment, vol. 113, no. 2, pp. 357-370, 2009.

[23] G. J. Morris, S. Naidu, and A. K. Topham, "Differences in breast carcinoma characteristics in newly diagnosed AfricanAmerican and Caucasian patients: a single-institution compilation compared with the national cancer institute's surveillance, epidemiology, and end results database," Cancer, vol. 110, no. 4, pp. 876-884, 2007.

[24] M. S. Moran, Q. Yang, L. N. Harris, B. Jones, D. P. Tuck, and B. G. Haffty, "Long-term outcomes and clinicopathologic differences of African-American versus white patients treated with breast conservation therapy for early-stage breast cancer," Cancer, vol. 113, no. 9, pp. 2565-2574, 2008.

[25] M. Sharma, J. D. Sharma, A. Sarma et al., "Triple negative breast cancer in people of North East India: Critical insights gained at a regional cancer centre," Asian Pacific Journal of Cancer Prevention, vol. 15, no. 11, pp. 4507-4511, 2014.

[26] G. H. Tan, N. A. Taib, W. Y. Choo et al., "Clinical characteristics of triple-negative breast cancer: experience in an Asian developing country," Asian Pacific Journal of Cancer Preventionv, vol. 10, pp. 395-398, 2009.

[27] C. Villarreal-Garza, J. N. Weitzel, M. Llacuachaqui et al., "The prevalence of BRCA1 and BRCA2 mutations among young Mexican women with triple-negative breast cancer," Breast Cancer Research and Treatment, vol. 150, no. 2, pp. 389-394, 2015.

[28] H. Kim and D. H. Choi, "Distribution of BRCA1 and BRCA2 mutations in asian patients with breast cancer," Journal of Breast Cancer, vol. 16, no. 4, pp. 357-365, 2013.

[29] R. H. Jack, E. A. Davies, C. Renshaw et al., "Differences in breast cancer hormone receptor status in ethnic groups: A London population," European Journal of Cancer, vol. 49, no. 3, pp. 696702, 2013.

[30] K. Lakshmaiah, U. Das, T. Suresh et al., "A study of triple negative breast cancer at a tertiary cancer care center in southern India," Annals of Medical and Health Sciences Research, vol. 4, no. 6, p. 933, 2014.

[31] V. G. Vogel, "Ongoing data from the breast cancer prevention trials: Opportunity for breast cancer risk reduction," $B M C$ Medicine, vol. 13, no. 1, article no. 63, 2015.

[32] J. Cuzick, I. Sestak, S. Cawthorn et al., “Tamoxifen for prevention of breast cancer: extended long-term follow-up of the IBISI breast cancer prevention trial," The Lancet Oncology, vol. 16, no. 1, pp. 67-75, 2015.

[33] V. G. Vogel, J. P. Costantino, D. L. Wickerham et al., "Effects of tamoxifen vs raloxifene on the risk of developing invasive breast cancer and other disease outcomes: the NSABP Study of Tamoxifen and Raloxifene (STAR) P-2 trial," JAMA, vol. 295, pp. 2727-2741, 2006.

[34] L. C. Hartmann, A. C. Degnim, R. J. Santen, W. D. Dupont, and K. Ghosh, "Atypical hyperplasia of the breast-risk assessment and management options," New England Journal of Medicine, vol. 372, no. 1, pp. 78-89, 2015.

[35] W. D. Dupont and D. L. Page, "Risk factors for breast cancer in women with proliferative breast disease," The New England Journal of Medicine, vol. 312, no. 3, pp. 146-151, 1985.

[36] S. Li, J. Liu, Y. Yang et al., "Impact of atypical hyperplasia at margins of breast-conserving surgery on the recurrence of breast cancer," Journal of Cancer Research and Clinical Oncology, vol. 140, no. 4, pp. 599-605, 2014.

[37] R. G. Roetzheim, J.-H. Lee, W. Fulp et al., "Acceptance and adherence to chemoprevention among women at increased risk of breast cancer," Breast, vol. 24, no. 1, pp. 51-56, 2015.

[38] S. Pruthi, R. E. Heisey, and T. B. Bevers, "Chemoprevention for breast cancer," Annals of Surgical Oncology, vol. 22, no. 10, pp. 3230-3235, 2015.

[39] A. Bosch, P. Eroles, R. Zaragoza, J. R. Viña, and A. Lluch, "Triple-negative breast cancer: Molecular features, pathogenesis, treatment and current lines of research," Cancer Treatment Reviews, vol. 36, no. 3, pp. 206-215, 2010. 


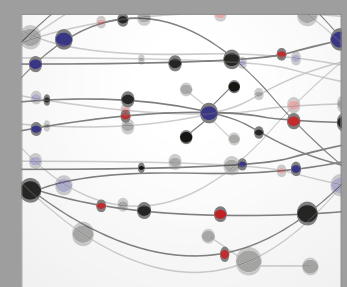

The Scientific World Journal
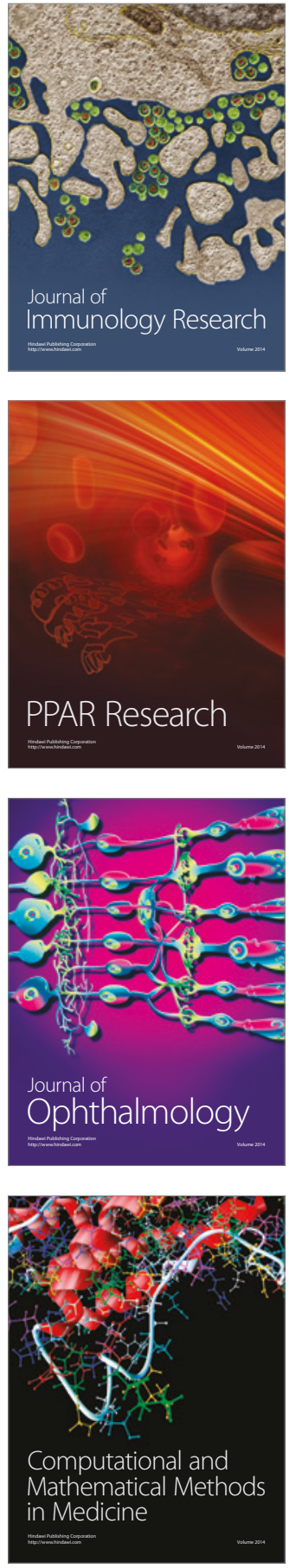

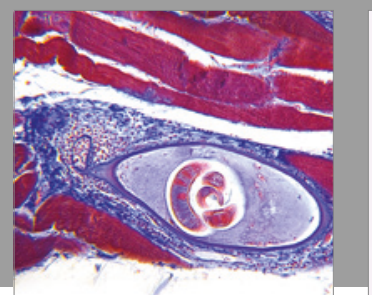

Gastroenterology Research and Practice
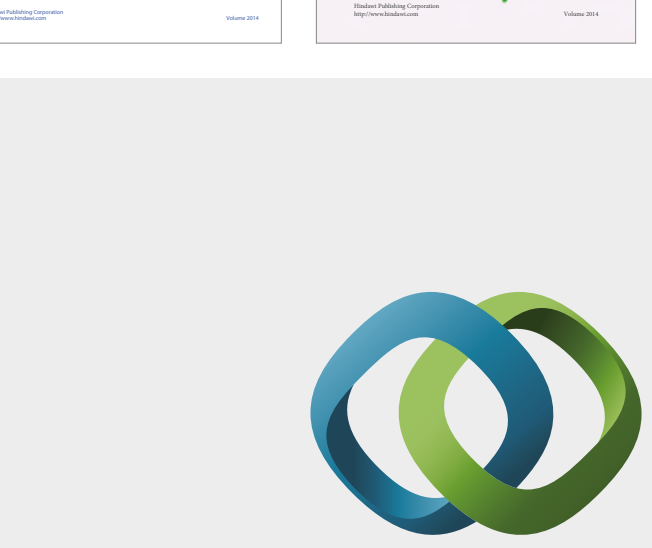

\section{Hindawi}

Submit your manuscripts at

https://www.hindawi.com
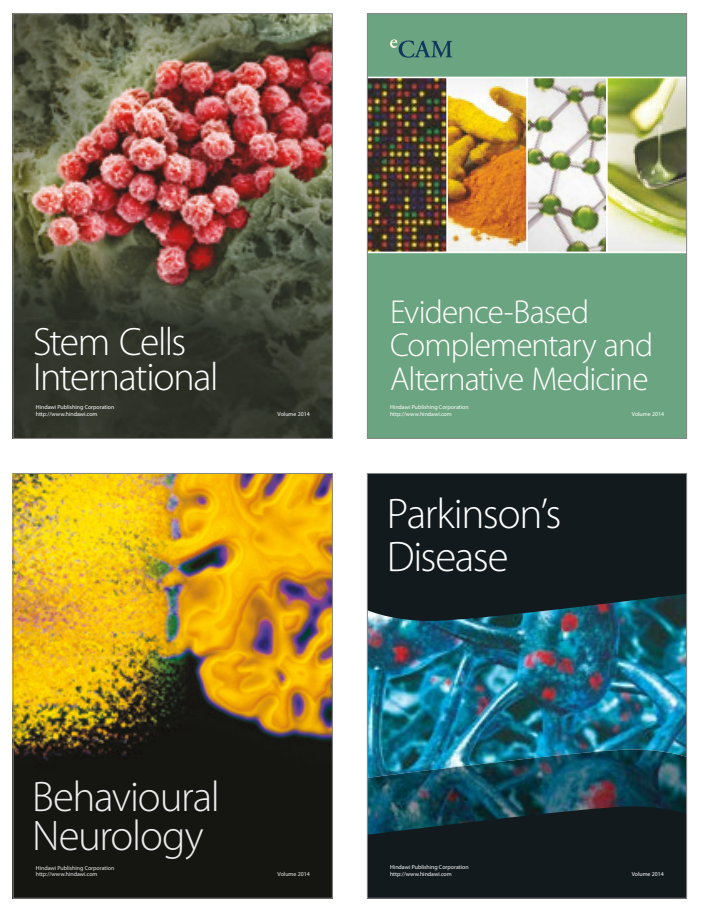
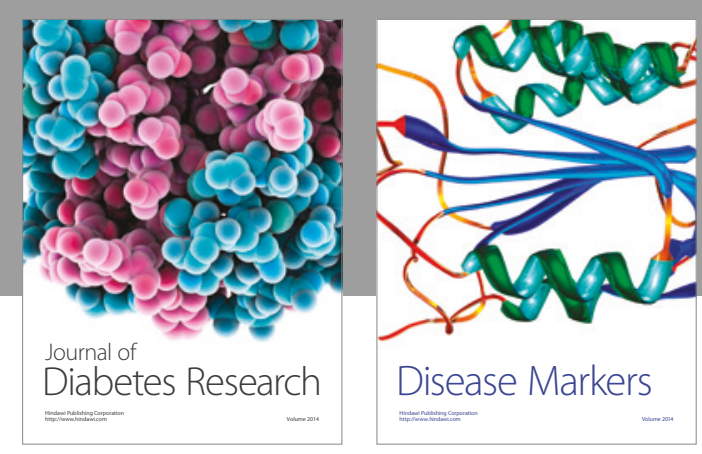

Disease Markers
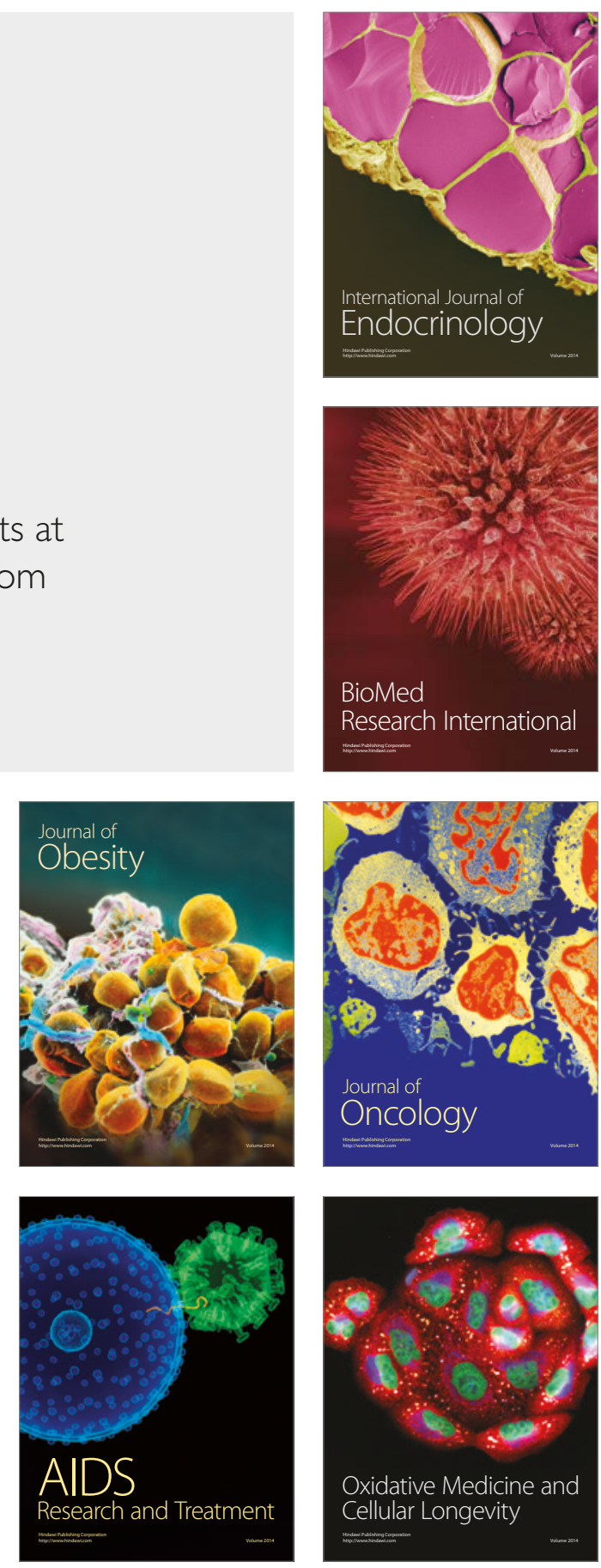